\title{
SN 2002kg - the brightening of LBV V37 in NGC 2403
}

\author{
K. Weis ${ }^{\star}$ and D. J. Bomans ${ }^{\star \star}$ \\ Astronomisches Institut, Ruhr-Universität Bochum, Universitätsstr. 150, 44780 Bochum, Germany \\ e-mail: kweis@astro.rub.de \\ Received 24 September 2004 / Accepted 13 November 2004

\begin{abstract}
SN $2002 \mathrm{~kg}$ is a type IIn supernova, detected in October 2002 in the nearby spiral galaxy NGC 2403 . We show that the position of SN $2002 \mathrm{~kg}$ agrees within the errors with the position of the LBV V37. Ground based and HST ACS images however show that V37 is still present after the SN $2002 \mathrm{~kg}$ event. We compiled a lightcurve of V37 which underlines the variablity of the object, and shows that SN $2002 \mathrm{~kg}$ was the brightening of V37 and not a supernova. The recent brightening is not a giant eruption, but more likely part of an S Dor phase. V37 shows strong $\mathrm{H}_{\alpha}+[\mathrm{N}$ II] emission in recent images and in the SN $2002 \mathrm{~kg}$ spectrum, which we interprete as the signature of the presence of an LBV nebula. A historic spectrum lacks emission, which may hint that we are witnessing the formation of an LBV nebula.
\end{abstract}

Key words. stars: evolution - stars: individual: V37 - stars: mass-loss - supernovae: individual: SN2002kg

\section{Introduction}

\section{1. $L B V s$ and their variations}

The most massive stars, above roughly $50 M_{\odot}$, pass an unstable phase as they turn into Luminous Blue Variable (LBVs). In the LBV phase, a transitional phase between the mainsequence and Wolf-Rayet state, the stars lose large amount of mass $\left(>10^{-5} M_{\odot} \mathrm{yr}^{-1}\right)$. As a consequence the evolution of these stars towards cooler temperature is stalled and reversed (Langer et al. 1994). The most massive stars seem not to enter the red supergiant phase (see van Genderen 2001 for a discussion). The coolest location of these stars in the Hertzsprung-Russell Diagram (HRD) marks the empirical Humphreys-Davidson limit (e.g. Humphreys \& Davidson 1994).

LBVs show variabilities on different timescales and with different strength (e.g. Spoon et al. 1994), the most classical being the S Dor variability (e.g. van Genderen et al. 1997a,b; van Genderen 2001). During an S Dor phase, which is a cyclic phenomenon of expansion and contraction at constant luminosity, the temperature decreases and rises, respectively. As a consequence the spectrum changes from a hot $\mathrm{O}$ or $\mathrm{B}$ type to a cooler mid A or early F type (Wolf 1992), so the colors become red and than blue again. It has been shown that the temperature variation in such a cycle is larger for more luminous LBVs (amplitude-luminosity-relation, Wolf 1989). Now, van Genderen (2001) subdivides the S Dor variability further in the short $S$ Dor phase, (S)-SD and the long $S$ Dor phase, $(L)$ $S D$. The (S)-SD is shorter than about 10 years, and the (L)-SD is larger than 20 years.

\footnotetext{
* Lise-Meitner fellow.

$\star \star$ Guest investigator of the UK Astronomy Data Centre.
}

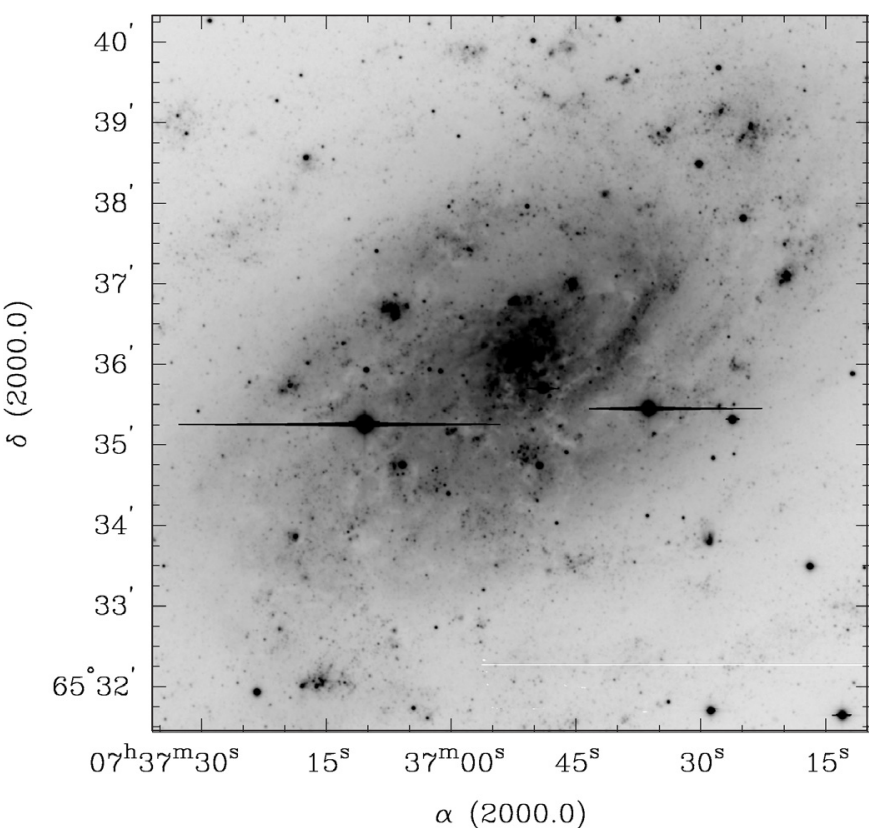

Fig. 1. A $g$-band image of NGC 2403 taken with the Isaac Newton telescope. A box indicates the area in which SN $2002 \mathrm{~kg}$ was found, and is shown as enlargement in Fig. 3.

More dramatic are the so called giant eruptions. These are spontaneous outbursts in which an LBV increases its luminosity by several magnitudes. It stays bright for a short time before rapidly declining back to lower luminosities, sometimes even lower as before the outburst. While becoming fainter their appearance becomes redder, presumably due to the formation of dust (e.g. Humphreys et al. 1999). The best known 


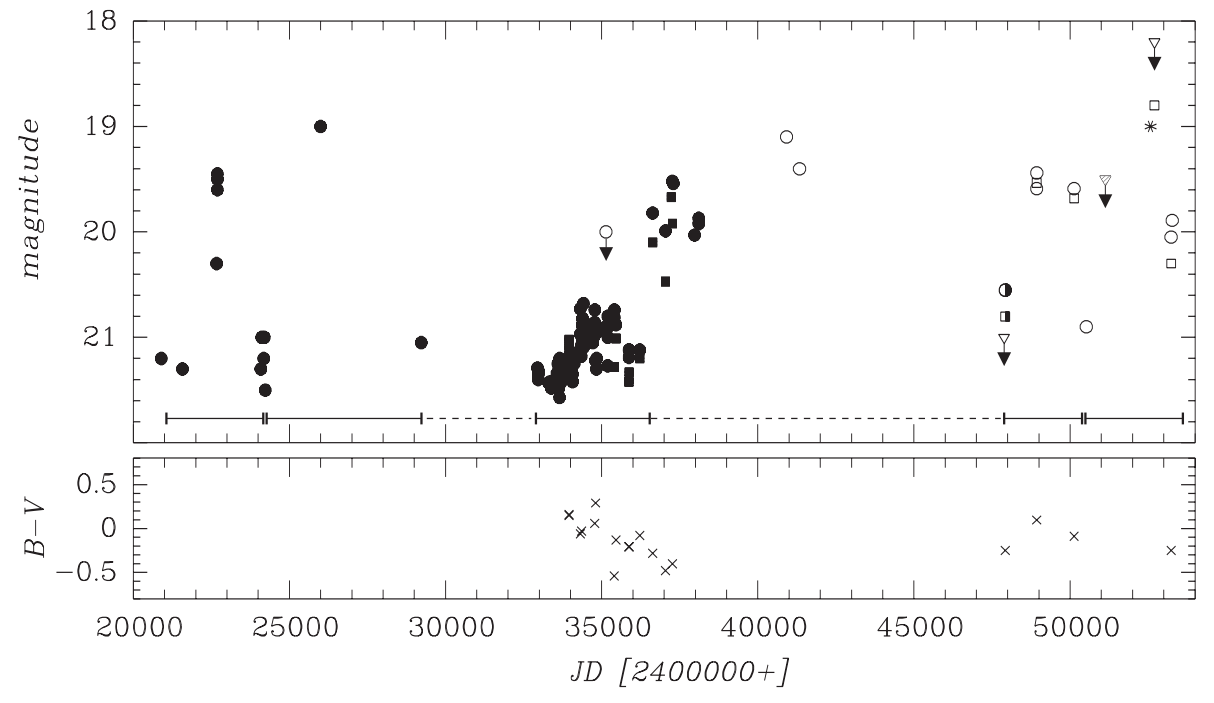

Fig. 2. The optical lightcurve of V37 (upper panel) and $B-V$ color (lower panel). Symbols are coded as: circles are $B$ magnitudes, squares are $V$ magnitudes, triangles are $R$ magnitudes. The detection of SN $2002 \mathrm{~kg}$ is indicated with a star, and is roughly an $R$ magnitude. Filled symbols are data taken from Tammann \& Sandage (1968), open symbols from this work, half-filled from Spiller (1992). The SN $2002 \mathrm{~kg}$ detection as well as one $R$ magnitude limit (hashed triangle) from Schwartz et al. (2003). Possible (S)-SD periods are indicated. example was $\eta$ Carinae's outburst around 1843, when it was with $-1^{\mathrm{m}}$ the second brightest star in the southern hemisphere (Innes 1903; Davidson \& Humphreys 1997). Other giant eruption LBVs are P Cygni ( 1600; de Groot 1988), SN1961V in NGC 1058 (1961; Goodrich et al. 1989) and SN1954J (=V12) in NGC 2403 (1954; Tammann \& Sandage 1968). These LBVs are called "giant eruption LBVs" or " $\eta$ Car Variables", or more recently "supernova impostors".

The high mass loss of LBVs and the ejecta of mass during the giant eruption leads to the formation of nebulae around LBVs (Nota et al. 1995; Weis 2001). These nebula are generally small $(<2 \mathrm{pc})$ but their expansion velocities show a large range. Several expand slowly $\left(\sim 30 \mathrm{~km} \mathrm{~s}^{-1}\right)$ others can be as fast as $100 \mathrm{~km} \mathrm{~s}^{-1}$ (Weis 2003). The fastest expansion velocities detected are in $\eta$ Carinae with $600 \mathrm{~km} \mathrm{~s}^{-1}$ (Homunculus) to at least $2500 \mathrm{~km} \mathrm{~s}^{-1}$ (outer ejecta) (e.g. Weis et al. 2001, 2004). LBV nebulae do show strong nitrogen emission due to the CNO processed material (Garcia-Segura et al. 1996).

\subsection{SN2002kg and V37 in NGC 2403}

SN $2002 \mathrm{~kg}$ was detected (Schwartz et al. 2003) in an unfiltered image from October 26, 2002 with the Katzman Automatic Imaging Telescope at Lick observatory (KAIT). SN $2002 \mathrm{~kg}$ is situated in NGC 2403 (see Fig. 1) a SBc spiral in the M 81 group (distance modulus $=28.14$, Tully 1988). At the time of discovery SN $2002 \mathrm{~kg}$ had a brightness of $19^{\mathrm{m}}$ in the unfiltered image. A spectrum of the SN 2002kg taken on January 6, 2003 taken with the Keck telescope identified SN 2002kg as type IIn, since it showed for a supernova quite narrow Balmer lines $\left(<500 \mathrm{~km} \mathrm{~s}^{-1}\right)$, casting already some doubt on being a classical supernova. Additionally, broader components ( $F W H M \sim$ $2500 \mathrm{~km} \mathrm{~s}^{-1}$ ) were also present. Unusual was the detection of [N II] emission at $6548 \AA$ and $6584 \AA$.

V37 has first been identified as a bright blue irregular variable by Tammann \& Sandage (1968), together with V12 (alias SN1954J), V22,V35, V38, today confirmed LBVs (Humphreys \& Davidson 1994). The lightcurve given in Fig. 2 shows the irregular behavior of V37, the original data of Tammann \& Sandage (1968) are included (filled symbols). From the

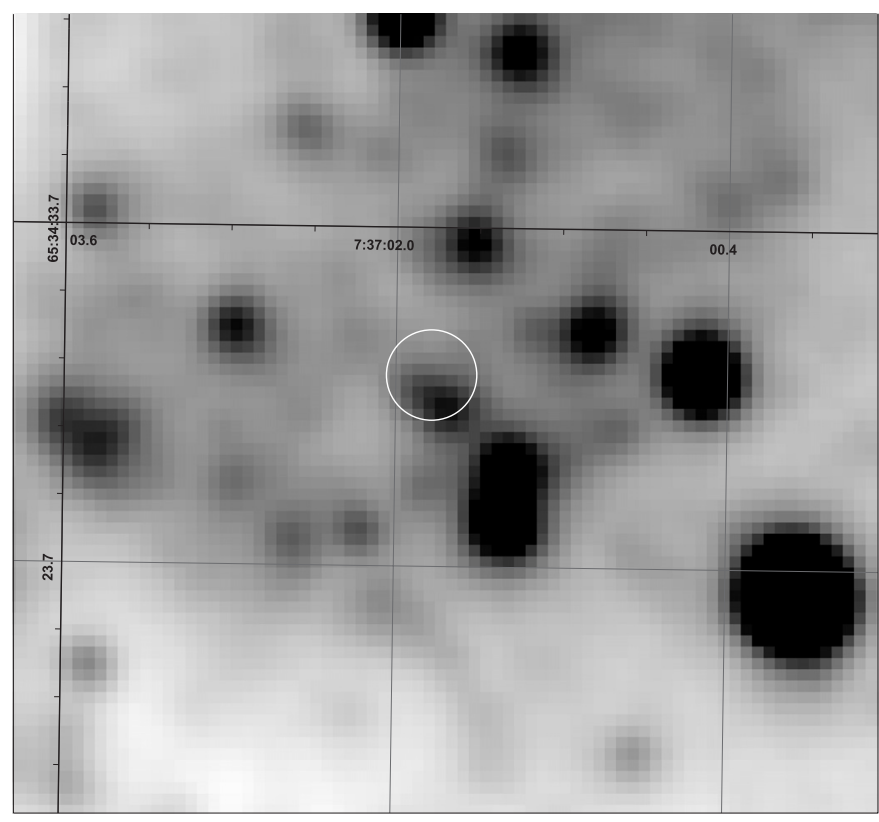

Fig. 3. This enlargement of Fig. 1 shows the closer vicinity and position (error circle) of SN 2002kg in NGC 2403. The image was taken in with the INT in 2001, before the detection as SN $2002 \mathrm{~kg}$.

brightness and colors Tammann \& Sandage (1968) estimated that these stars are F supergiants. A spectrum of V37 obtained in 1985 shows according to Humphreys \& Aaronson (1987) absorption-lines, a blue continuum, and no emission lines.

\section{Observations and analysis}

\subsection{Astrometry}

The position of SN $2002 \mathrm{~kg}$ as given at discovery (Schwartz et al. 2003) is $\alpha=7 \mathrm{~h} 37 \mathrm{~m} 1.83 \mathrm{~s}$ and $\delta=+65^{\circ} 34^{\prime} 29^{\prime \prime} 3$ (2000.0). We used a deep INT archival image in the $g$-band to investigate the surroundings of SN $2002 \mathrm{~kg}$. Using astrometry routines in IRAF/STSDAS and KARMA we transferred the coordinate system of the DSS image onto the CCD frame. Given an uncertainty of the process and the not corrected higher 


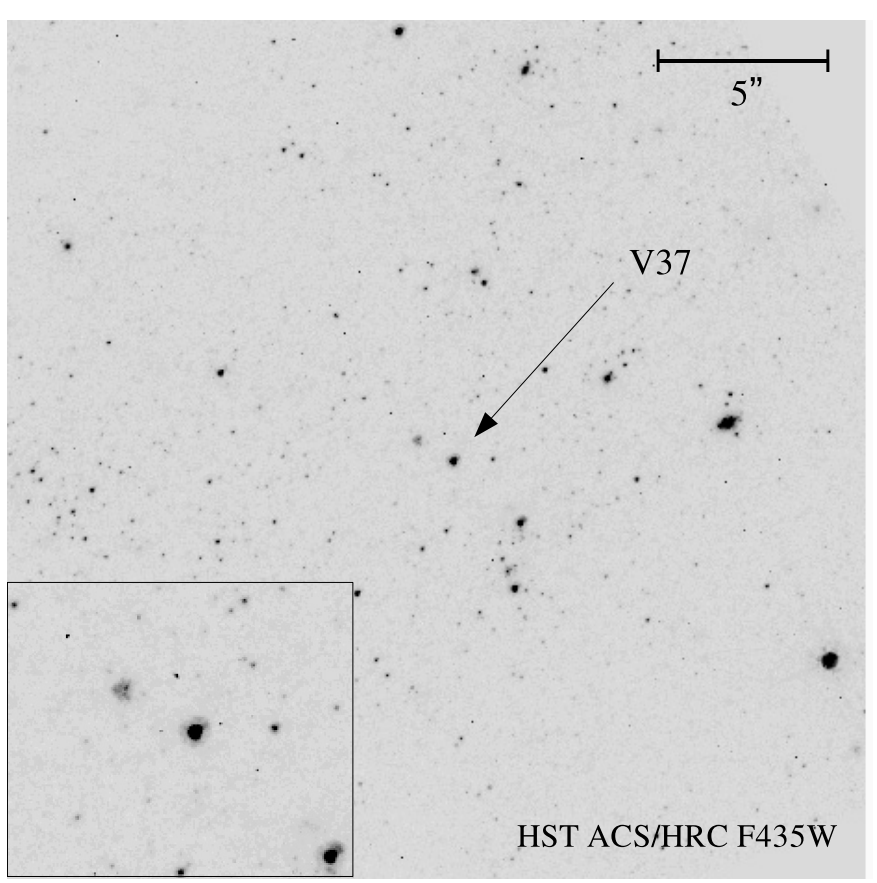

Fig. 4. HST ACS/HRC image in the $F 435 W(\sim B)$ image shown the same section as in Fig. 3. The area of V37 is shown as enlargement in the lower left corner again.

order distortions of the CCD we reach an absolute positioning of slightly better than $1^{\prime \prime}$. Assuming a similar accuracy for the position of SN $2002 \mathrm{~kg}$ we generated Fig. 3, with the $g$-band image in gray-scale and the error circle overlayed. It shows clearly, that SN2002kg is coincident with the stellar source identified as V37 by Tammann \& Sandage (1968).

\subsection{Ground based photometry}

For the construction of a lightcurve (Fig. 2) we used the measurements by Tammann \& Sandage (1968) as starting point. The $B, V$ photometry also provided us with a decent number of secondary photometric standard stars in the field of NGC 2403. We uses these stars to tie our photometry made on a large variety of images to Tammann-Sandage's system. We ignore color terms between the photographic photometry and our CCD images in most cases. For the few very good data we verified that the effect is generally smaller than the photometric uncertainties due to crowding, seeing, and centering of the aperture. All measurements where done with IRAF/DAOPHOT through Tammann-Sandage's 6!'7 aperture. We measured the DSS, DSS2-blue, and DSS2-red (1955, 1997, 1989), as well as two blue plates from the Tautenburg $2 \mathrm{~m}$ Schmidt (from 1970 and 1972), several CCD images from the Isaac Newton $2.5 \mathrm{~m}$ (1992, 1996, 2001), Jacobus Kapteyn 1m telescopes (1996, 1998) retrieved from the ING archive, and CCD images from the Tautenburg $2 \mathrm{~m}(2003,2004)$. Whenever the object is near or below detection limit, we also estimated the magnitude (or magnitude limit) in comparison to the stars of secondary standard sequence in the traditional way by eye. For comparison we also added the measurements reported in the discovery IAUC of SN 2002kg (Schwartz et al. 2003). These measurements where taken with an unfiltered CCD mounted at the KAIT. We converted them into $R$ band measurements following the recipe described in Li et al. (2003).

\subsection{HST ACS images}

Very recently HST ACS/WFC and ACS/HRC images of NGC 2403 were taken, which have the region V37 in the field of view. We retrieved WFC images in the $F 475 W, F 606 W$, $F 814 W$, and $F 658 N$ filters, and HRC images in the $F 435 W$, and $F 625 W$ filters from the HST archive. V37 is present on the images as a blue point source at exactly the position predicted based on the 2001 INT images. This clearly shows that SN $2002 \mathrm{~kg}$ was not a SN event, but a brightening of V37. We measured the brightness in the ACS $B$ and $V$ bands and added the points to our lightcurve. These data points show that V37 is fading again and of quite blue color. The HRC images (Fig. 4) also reveal that next brightest (fainter by $1.6^{\mathrm{m}}$ in $F 475 \mathrm{~W}$ ) object in the positional error circle is not a star but a blue diffuse object of uncertain nature (see enlarged box).

\section{Discussion and conclusions}

SN $2002 \mathrm{~kg}$ was from the detection on a very strange SN IIn. Being quite faint at detection (about $-9^{\mathrm{m}}$, absolute) and with an unusual spectrum it was suspected that SN 2002kg may not be a real supernova. Unfortunately, its lightcurve was not monitored. A comparison of ground based images (Fig. 3) taken before the supernova event with the position of SN 2002kg shows two sources, a diffuse object and a bright one, the latter being the LBV V37. Astrometry on the ground based and HST ACS images showed that the bright star is indeed coincident with SN $2002 \mathrm{~kg}$. The HST ACS images, as well as images from Tautenburg show both sources present after 2002, which clearly proves that SN $2002 \mathrm{~kg}$ was rather the brightening of a luminous star, historically known as V37. The diffuse source with a size of $0.18 F W H M(3.7 \mathrm{pc})$ is too large for being a young SNR (created 2002). The source is also present in our best archival images before 2002. It is also too faint for a tight cluster of massive stars, which could be progenitors of a type IIn supernova. The SN $2002 \mathrm{~kg}$ could be a supernova in a more distant background galaxy, but than the velocity of the emission lines detected (Schwartz et al. 2003) would be different, and this is not the case.

The lightcurve of V37 in Fig. 2 shows several possible cycles of 2000-3000 days period during the last 80 yrs as indicated in Fig. 2. This is consistent with (S)-SD type variations. However the brightening around MJD 35000 shows a change towards bluer colors. This is unusual as S Dor variabilities normally show a redward color trend. Such an increase in brightness with bluer colors is rarely seen in LBVs, with the exceptions being presently $\eta$ Carinae (Whitelock et al. 2004), and NGC $2366 \mathrm{V1}$, which became UV brighter during its $V$-band fading (Drissen et al. 2001). There is no indication for a giant eruption in the lightcurve of V37, but there are still large time gaps which in theory could accommodate such an event in the past. 


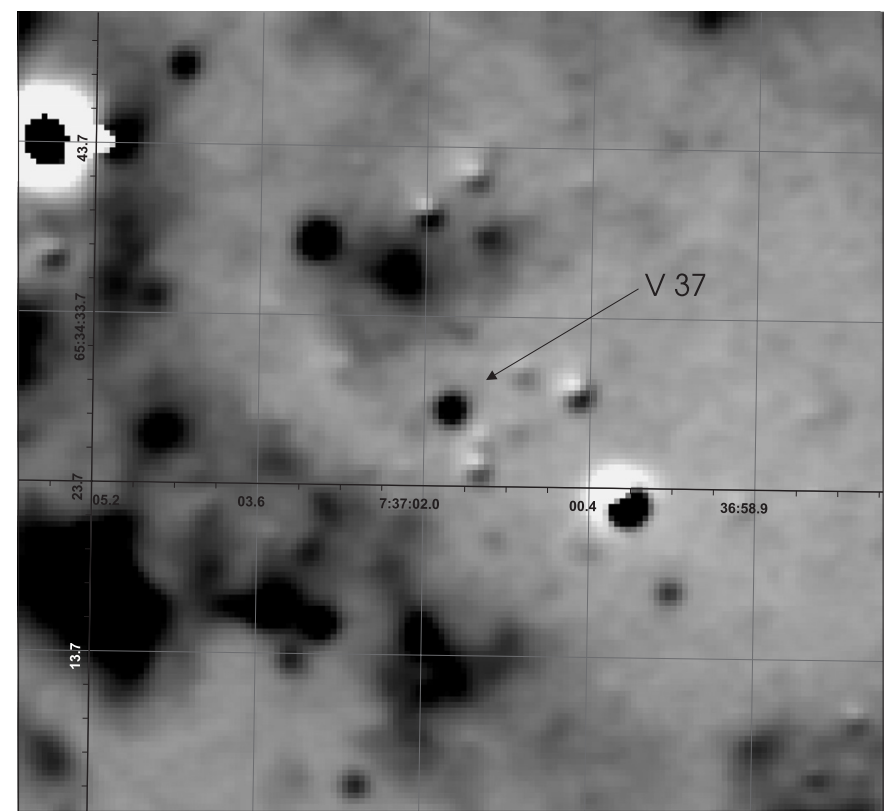

Fig. 5. This $\mathrm{H}_{\alpha}$ emission line image generated from INT images taken in 2001, indicates that V37 is an $\mathrm{H}_{\alpha}$ bright source.

With our data sets we could generate two continuum corrected $\mathrm{H}_{\alpha}$ images of the region around V37. In 2001 V37 is a bright $\mathrm{H}_{\alpha}$ emitter (see Fig. 5). The HST ACS image from 2004 taken in the F658N filter also shows bright emission at the position of V37 after correction for the continuum emission. The spectrum of SN $2002 \mathrm{~kg}$ (Sect. 1.2) indicates that $\mathrm{H}_{\alpha}$ and [N II] emission is present at that time. One possible explanation is the creation of an LBV nebula around V37 coinciding with the recent bright phase. Such a nebula would show strong nitrogen emission and could expand with velocities as high as detected in the SN 2002kg spectrum, see Sect. 1.1. The H II region [H83] 177 identified earlier by Hodge \& Kennicutt (1983) on a deep $\mathrm{H}_{\alpha}+[\mathrm{N}$ II $]$ plate seems to coincide within the errors with the position of V37, too. That could indicate that the star has been emitting $\mathrm{H}_{\alpha}$ and maybe [N II] as early as the 1980s, which would point at an earlier nebula formation. Still, missing emission lines in the spectrum of Humphreys \& Aaronson (1987) could mean, that the $\mathrm{H}_{\alpha}$ emission would just indicate a strong stellar and variable $\mathrm{H}_{\alpha}$ line. The missing [N II] lines in that spectrum may imply that the nebula has not been formed in 1985. Whether there is nebula formation during earlier times or not, may only be answered with the detection (or high quality non-detection) of [N II] lines in other historic spectra. In any case, it seems, that $\mathrm{V} 37$ has a nitrogen enhanced nebula now.

In V37 we may witness currently the creation of an LBV nebula, but it is not yet clear whether this nebula was created during a giant eruption, as shell ejection during several SD periods as in P Cyg (e.g. Markova et al. 2001), or recently in connection with the brightening in 2002. Determining the exact evolutionary state of V37 is therefore of importance for our understanding of the LBV phenomenon and very massive stars in general.
Acknowledgements. K.W. is supported by the state of North Rhine-Westphalia (Lise-Meitner fellowship). We thank S. Klose for kindly providing his Tautenburg $2 \mathrm{~m}$ images, and $\mathrm{H}$. Meusinger for scanning the historic Tautenburg plates. We thank the referee A. M. van Genderen for his comments that helped to significantly improve the paper. This research is partially based on data from the ING Archive. Based partly on observations made with the NASA/ESA Hubble Space Telescope, obtained from the data archive at the Space Telescope Institute. This research has made use of NASA's Astrophysics Data System.

\section{References}

Davidson, K., \& Humphreys, R. M. 1997, ARA\&A, 35, 1

de Groot, M. 1988, Irish Astron. J., 18, 163

Drissen, L., Crowther, P. A., Smith, L. J., et al. 2001, ApJ, 546, 484

Garcia-Segura, G., Mac Low, M.-M., \& Langer, N. 1996, A\&A, 305, 229

Goodrich, R. W., Stringfellow, G. S., Penrod, G. D., \& Filippenko, A. V. 1989, ApJ, 342, 908

Hodge, P. W., \& Kennicutt, R. C. 1983, AJ, 88, 296

Humphreys, R. M., \& Aaronson, M. 1987, AJ, 94, 1156

Humphreys, R. M., \& Davidson, K. 1994, PASP, 106, 1025

Humphreys, R. M., Davidson, K., \& Smith, N. 1999, PASP, 111, 1124

Innes, R. T. A. 1903, Ann. Cape Obs., 9, 75B

Langer, N., Hamann, W.-R., Lennon, M., et al. 1994, A\&A, 290, 819

Li, W., Filippenko, A. V., Chornock, R., \& Jha, S. 2003, PASP, 115, 844

Markova, N., Morrison, N., Kolka, I., \& Markov, H. 2001, A\&A, 376, 898

Nota, A., Livio, M., Clampin, M., \& Schulte-Ladbeck, R. 1995, ApJ, 448,788

Schwartz, M., Li, W., Filippenko, A. V., \& Chornock, R. 2003, IAU Circ., 8051, 1

Spiller, F. 1992, Ph.D. Thesis, Universität Heidelberg

Spoon, H. W. W., de Koter, A., Sterken, C., Lamers, H. J. G. L. M., \& Stahl, O. 1994, A\&AS, 106, 141

Tammann, G. A., \& Sandage, A. 1968, ApJ, 151, 825

Tully, R. B. 1988, Nearby galaxies catalog (Cambridge and New York:

Cambridge University Press), 221

van Genderen, A. M. 2001, A\&A, 366, 508

van Genderen, A. M., de Groot, M., \& Sterken, C. 1997a, A\&AS, 124, 517

van Genderen, A. M., Sterken, C., \& de Groot, M. 1997b, A\&A, 318, 81

Weis, K. 2001, Rev. Mod. Astron., 14, 261

Weis, K. 2003, A\&A, 408, 205

Weis, K., Corcoran, M. F., Bomans, D. J., \& Davidson, K. 2004, A\&A, 415, 595

Weis, K., Corcoran, M. F., \& Davidson, K. 2001, in Astronomische Gesellschaft Meeting Abstracts, 211

Whitelock, P. A., Feast, M. W., Marang, F., \& Breedt, E. 2004, MNRAS, 352, 447

Wolf, B. 1989, A\&A, 217, 87

Wolf, B. 1992, Rev. Mod. Astron., 5, 1 